\title{
Dual-energy spectral CT quantitative parameters for the differentiation of Glioma recurrence from treatment-related changes: a preliminary study
}

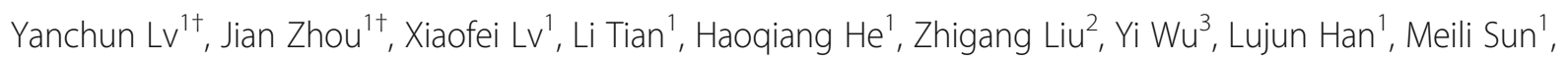
Yadi Yang ${ }^{1}$, Chengcheng Guo ${ }^{4}$, Cong Li ${ }^{4}$, Rong Zhang ${ }^{1}$, Chuanmiao Xie', Yinsheng Chen ${ }^{4^{*+}}$ and Zhongping Chen ${ }^{4^{*}+}$ (D)

\begin{abstract}
Background: Differentiating glioma recurrence from treatment-related changes can be challenging on conventional imaging. We evaluated the efficacy of quantitative parameters measured by dual-energy spectral computed tomographic (CT) for this differentiation.

Methods: Twenty-eight patients were examined by dual-energy spectral $C T$. The effective and normalized atomic number $\left(Z_{\text {eff }}\right.$ and $Z_{\text {eff-N }}$, respectively); spectral Hounsfield unit curve $\left(\lambda_{H U}\right)$ slope; and iodine and normalized iodine concentration (IC and $I_{N}$, respectively) in the post-treatment enhanced areas were calculated. Pathological results or clinicoradiologic follow-up of $\geq 2$ months were used for final diagnosis. Nonparametric and $t$-tests were used to compare quantitative parameters between glioma recurrence and treatment-related changes. Sensitivity, specificity, positive and negative predictive values (PPV and NPV, respectively), and accuracy were calculated using receiver operating characteristic (ROC) curves. Predictive probabilities were used to generate ROC curves to determine the diagnostic value.
\end{abstract}

Results: Examination of pre-contrast $\lambda_{H U}, Z_{\text {eff, }} Z_{\text {eff-N, }}, I C, I C_{N}$, and venous phase $I C_{N}$ showed no significant differences in

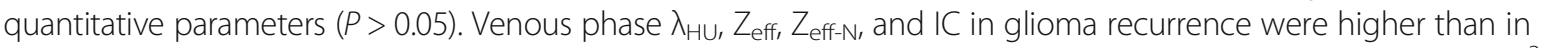
treatment-related changes $(P<0.001)$. The optimal venous phase threshold was $1.03,7.75,1.04$, and $2.85 \mathrm{mg} / \mathrm{cm}^{3}$, achieving 66.7, 91.7, 83.3, and 91.7\% sensitivity; 100.0, 77.8, 88.9, and 77.8\% specificity; 100.0, 73.3, 83.3, and 73.3\% PPV; 81.8, 93.3, 88.9, and 93.3\% NPV; and 86.7, 83.3, 86.7, and 83.3\% accuracy, respectively. The respective areas under the curve (AUCS) were $0.912,0.912,0.931$, and 0.910 in glioma recurrence and treatment-related changes.

Conclusions: Glioma recurrence could be potentially differentiated from treatment-related changes based on quantitative values measured by dual-energy spectral CT imaging.

Keywords: Glioma, Dual energy spectral CT, Recurrence

\footnotetext{
* Correspondence: chenyinsh@sysucc.org.cn; chenzhp@sysucc.org.cn

†Yanchun Lv and Jian Zhou contributed equally to this work and share the first authorship.

'Zhongping Chen and Yingsheng Chen contributed equally to this work and

share the corresponding authorship.

${ }^{4}$ Department of Neurosurgery/Neuro-oncology, Sun Yat-sen University

Cancer Center, State Key Laboratory of Oncology in South China,

Collaborative Innovation Center for Cancer Medicine, 651 Dongfeng Road

East, Guangzhou 510060, China

Full list of author information is available at the end of the article
}

(c) The Author(s). 2020 Open Access This article is distributed under the terms of the Creative Commons Attribution 4.0 International License (http://creativecommons.org/licenses/by/4.0/), which permits unrestricted use, distribution, and

reproduction in any medium, provided you give appropriate credit to the original author(s) and the source, provide a link to the Creative Commons license, and indicate if changes were made. The Creative Commons Public Domain Dedication waiver (http://creativecommons.org/publicdomain/zero/1.0/) applies to the data made available in this article, unless otherwise stated. 


\section{Background}

Differentiation between glioma recurrence and treatmentrelated changes (necrosis after operation or radiation, pseudoprogression after chemotherapy) remains a significant challenge. Clinically, the two entities have totally different consequences; however, both often share the same symptoms and show very similar features in conventional magnetic resonance imaging (MRI) and computed tomography $(\mathrm{CT})[1,2]$. Given that the management strategies for tumor recurrence and treatment-related changes are completely distinct, it is crucial for clinicians to be able to differentiate these outcomes [3].

Many advanced imaging techniques such as functional magnetic resonance imaging (fMRI), positron emission tomography (PET), and single photon emission CT (SPECT) have been used in an attempt to distinguish these two conditions. These techniques, however, are imperfect, and accurate differentiation of treatmentrelated changes remains difficult [2-8].

In 2011, a novel spectral CT method known as gemstone spectral imaging (GSI) was introduced; GSI uses dual energy $\mathrm{X}$-rays produced by the rapid switching of low $(80 \mathrm{kVp})$ and high $(140 \mathrm{kVp})$ tube voltages [9]. Quantitative parameters measured on GSI have been used to diagnose several tumor types [9-13].
Herein, we explored the use of quantitative parameters measured by dual-energy GSI-CT to differentiate between glioma recurrence and treatment-related changes.

\section{Methods \\ Patients}

The ethics committee at Sun Yat-sen University Cancer Center approved this retrospective study; all included patients provided informed consent. In all, 28 patients (13 men and 15 women; mean age: $39.3 \pm 13.0$ years) who underwent brain dual-energy GSI-CT were enrolled. All patients had undergone surgery for tumor removal, and the inclusion criteria were as follows: (1) histologically confirmed glioma; (2) the primary treatments were surgery, chemotherapy (temozolomide), or radiation therapy (total received dose: 40-60 Gy); and (3) detectable subsequently developed new contrastenhanced lesions. Exclusion criteria were defined as definite contraindications for contrast-agent administration, cardiopathy, or pregnancy. The final diagnosis was determined based on either a second surgery or a followup examination. The follow-up evaluation was conducted at intervals of $\geq 2$ months. In the case of followup diagnoses, treatment-related changes were confirmed in the event of complete disappearance of the enhancing

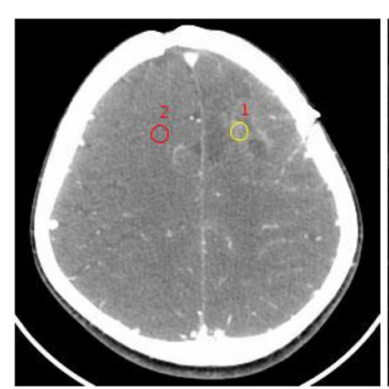

a

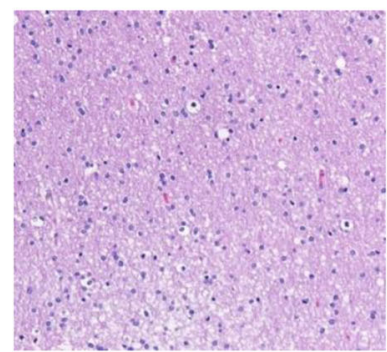

d

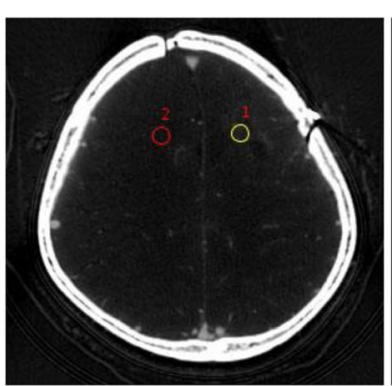

b

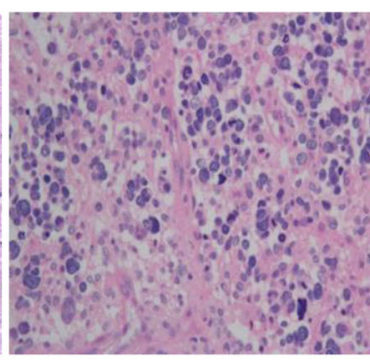

e

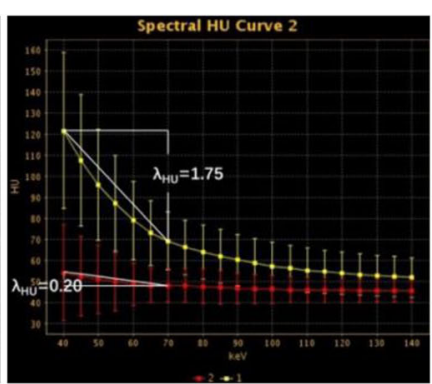

c

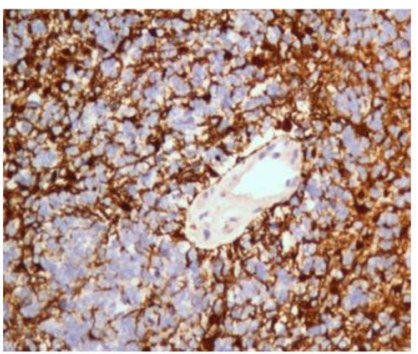

f

Fig. 1 Contrast-enhanced venous phase GSI images show that IC and spectral curve were significantly different in glioma recurrence and the normal reference brain parenchyma. a Contrast-enhanced 70-keV monochromatic image (L1: area, $54.16 \mathrm{~mm} 2$; mean CT value, 69.33 HU; L2: $54.16 \mathrm{~mm} 2$; mean $\mathrm{CT}$ value, $48.06 \mathrm{HU})$. $\mathbf{b}$ lodine-based materialdecomposition. image shows that IC in glioma recurrence and the normal reference brain parenchyma were $0.915 \mathrm{mg} / \mathrm{cm} 3$. and $0.113 \mathrm{mg} / \mathrm{cm} 3$ (L1: area, $54.16 \mathrm{~mm} 2 ;$ mean IC, $9.15 \cdot 100 \mu \mathrm{g} / \mathrm{cm} 3 ; \mathrm{L} 2:$ area, $54.16 \mathrm{~mm} 2 ; \mathrm{mean}$ IC, $1.13 \cdot 100 \mathrm{\mu g} / \mathrm{cm} 3)$. c Graph shows spectral HU curve of glioma recurrence (yellow) and the normal reference brain parenchyma (red), slope of the curve representing glioma recurrence is much higher than the normal reference brain parenchyma (1.75 vs. 0.20). $\mathbf{d}$ The pathology noted after the first operation indicated astrocytoma (Grade II). e A large of tumor cells showed diffused distribution in the smear; eosinophil, nuclear were marked atypia, and the pathologic diagnosis was glioblastoma (Grade IV). f The GFAP was positive 
lesion, partial resolution, if stable on subsequent followup images over a minimum period of 2 months, or if the patient was in a stable clinical state and showed no new neurologic symptoms. The glioma recurrence was based on the development of neurologic symptoms and a progressive increase in the size of the enhancing lesion or a new enhancing lesion on follow-up examination. Magnetic resonance imaging (MRI) enhancements or MR spectroscopy (MRS) were also used to help define treatment-related changes or glioma recurrence. All images were assessed in consensus by two radiologists (YL and JZ) with 20 and 8 years of experience in radiology, respectively.

\section{Dual energy gemstone spectral $\mathrm{CT}$ examination}

The Discovery CT750HD scanner (GE Healthcare, Waukesha, WI, US) was used for scanning. The following scanning parameters in the GSI mode were used: tube voltage of $140 \mathrm{kV}$ and $80 \mathrm{kV}$ and $0.5-\mathrm{ms}$ instantaneous switch; tube current, 0-600 mA automatic modulation; collimation thickness, $0.625 \mathrm{~mm}$; rotation speed, $0.8 \mathrm{~s}$; and helical pitch, 1.375 . The total CT dose index volume used in this study was $18.28 \mathrm{mGy}, 69.5 \%$ lower than the CT dose index volume of $59.89 \mathrm{mGy}$ used for average conventional head scanning at our institution. An automated injector was used to inject an iodinated nonionic contrast agent (iopamidol 300; Bracco, Milan, Italy) at $2.8 \mathrm{~mL} / \mathrm{s}$ and $1.5 \mathrm{~mL} / \mathrm{kg}$ through the right ulnar vein. The scan's venous phase delay time was $50 \mathrm{~s}$.

\section{Acquisition of GSI quantitative parameters}

The GSI viewer 4.5 (GE Healthcare) was used to acquire GSI images. The region of interest (ROI) was plotted on the pre-contrast scan and the reconstructed monochromatic venous phase data images on $70 \mathrm{keV}$. The ROI was targeted for most suspicious areas of tumor recurrence with nodular enhancement, with care to exclude calcification and minute vessel. The same ROI was copied on the other common brain parenchyma as a contrast. The CT-based effective atomic number $\left(\mathrm{Z}_{\text {eff }}\right)$ and iodine concentration (IC) values in monochromatic images and iodine-based material-decomposition images for each ROI were automatically calculated (Figs. 1a, b and 2a, b). All ROIs were automatically copied on all

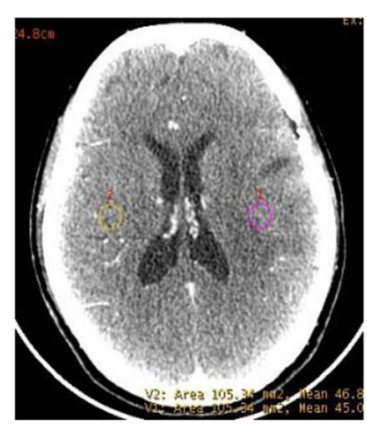

a

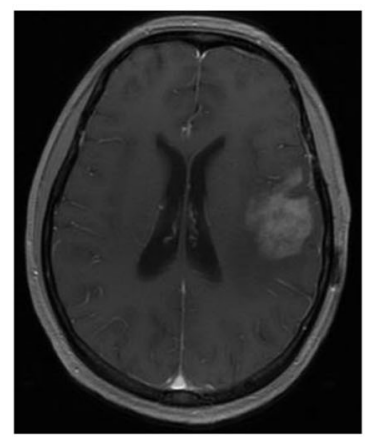

d

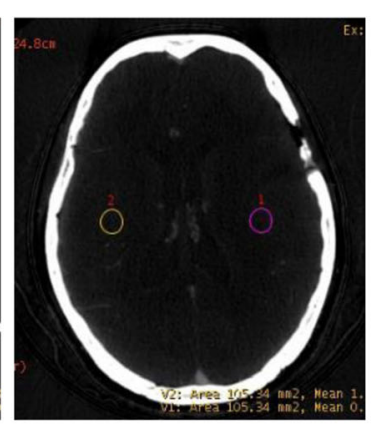

b

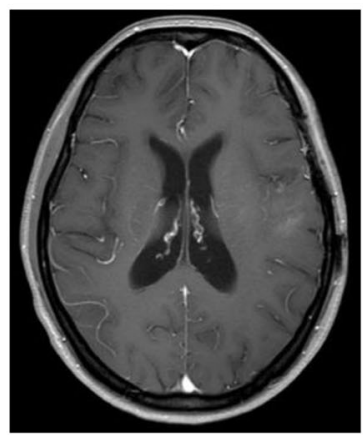

e

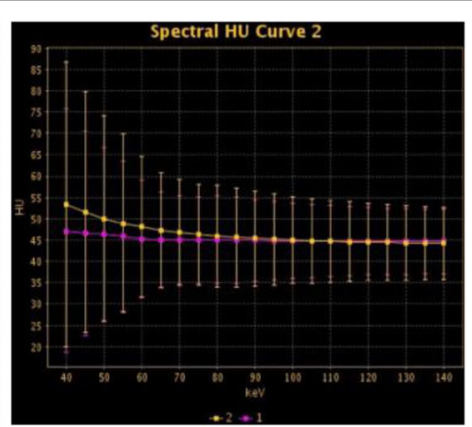

C

Fig. 2 Contrast-enhanced venous phase GSI images show that IC and spectral curve were similar in treatment related necrosis and the normal reference brain parenchyma. a Contrast-enhanced 70-keV monochromatic image (L1: area, 105.34 mm2; mean CT value, 45.01 HU; L2: 105.34 mm2; mean $C T$ value, $46.8 \mathrm{HU}$ ). b lodine-based materialdecomposition image shows that IC in glioma recurrence and the normal reference brain

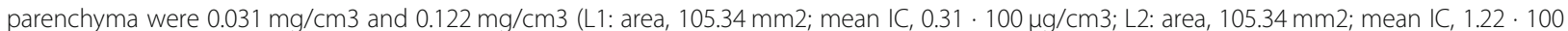
$\mu \mathrm{g} / \mathrm{cm} 3)$. c Graph shows spectral HU curve of glioma recurrence (violet) and the normal reference brain parenchyma yellow), slope of the curve representing glioma recurrence is similar with the normal reference brain parenchyma (0.07 vs. 0.22). d The same time with dual energy gemstone spectral CT scanning MRI T1WI enhanced image showed recurrence treatment related necrosis. e Seven months later, the MRI T1WI enhanced image showed the treatment related necrosis was obviously small with slight enhancement 
monochromatic images and iodine-based materialdecomposition images. All measurements were independently obtained by two radiologists.

\section{Data processing and statistical analysis}

$\mathrm{Z}_{\mathrm{eff}}$, IC (in $\mathrm{mg} / \mathrm{mL}$ ), and $\mathrm{CT}$ values on monochromatic images $(40-140 \mathrm{keV})$ were calculated and exported by the average values of two radiologists. The $Z_{\text {eff }}$ of the glioma $\left(\mathrm{Z}_{\text {eff-gli }}\right)$ and $\mathrm{IC}$ of the glioma $\left(\mathrm{IC}_{\mathrm{gli}}\right)$ were normalized to values in the normal reference brain parenchyma $\left(\mathrm{Z}_{\text {eff-Bp }}\right.$ and $\left.\mathrm{IC}_{\mathrm{BP}}\right)$ to obtain normalized $\mathrm{Z}_{\text {eff }}\left(\mathrm{Z}_{\text {eff-N }}\right)$ and IC $\left(\mathrm{IC}_{\mathrm{N}}\right): \quad \mathrm{Z}_{\text {eff-N }}=\mathrm{Z}_{\text {eff-gli }} / \mathrm{Z}_{\text {eff-BP }}$ and $\mathrm{IC}_{\mathrm{N}}=\mathrm{IC}_{\mathrm{gli}} / \mathrm{IC}_{\mathrm{BP}}$, where $\mathrm{BP}$ is the normal reference brain parenchyma. The Hounsfield unit curve slope $\left(\lambda_{\mathrm{HU}}\right)$ was indicated as the differences between the CT value on $40 \mathrm{keV}$ and 70 $\mathrm{keV}$ divided by the energy difference $(30 \mathrm{keV}): \lambda_{\mathrm{HU}}=(40$ $\mathrm{keV}_{\mathrm{HU}}-70 \mathrm{keV}_{\mathrm{HU}}$ )/30 keV (Fig. 1c and 2c).

Quantitative data were saved as means and standard deviation $\left({ }^{-} x \pm s\right)$ or medians with interquartile range. All the GSI quantitative parameters were compared by two independent samples $t$-test and nonparametric tests. Predictive probabilities were used to generate ROC curves to evaluate the diagnostic value. Further, accuracy, positive predictive value (PPV), and negative predictive value (NPV) were calculated. The maximum Youden's index value was chosen as the best threshold. Data were analyzed using statistical software package (SPSS version 21.0; SPSS Inc., IBM Corp, NY). $P<0.05$ was considered to be statistically significant.

\section{Results}

\section{Clinical and pathological results}

In all, 28 patients were examined with dual energy gemstone spectral CT. Fifteen women [mean age, $36.9 \pm 10.6$ years] and 13 men [mean age, $42.2 \pm 15.3$ years] were included in the final analysis. A total of 30 lesions (12 glioma recurrence lesions, 18 treatment-related change lesions) were enrolled for evaluation.

The primary histopathology as per WHO 2007 classification was 15 Grade II (53.6\%), 7 Grade III (25\%), 6 Grade IV (21.4\%). The primary histopathology was 6 glioblastomas (21.4\%), 8 astrocytomas $(28.6 \%), 3$ anaplastic astrocytomas (10.7\%), 2 oligodendrogliomas (7.1\%), 3 anaplastic oligodendrogliomas (10.7\%), 3 oligoastrocytomas (10.7\%), 2 anaplastic oligoastrocytomas (7.1\%), 1 ganglioglioma (3.6\%). The primary treatments were 3 operation only (10.7\%); 5 operation and radiation therapy $(17.9 \%) ; 20$ operation, radiation therapy, and chemotherapy (71.4\%).

Pathology after operation showed glioma recurrence in 5 patients ( 5 lesions) and treatment-related changes in 2 patients (2 lesions). The recurrence group of second histopathology showed 2 glioblastomas (Grade IV), 1 astrocytoma (Grade II), 1 anaplastic oligodendroglioma (Grade III), 1 and anaplastic oligoastrocytoma (Grade III).

Six patients (7 lesions) without pathologic evaluation were finally classified into the glioma recurrence group up to a median period of 5 months (range, 2-24 months). Fifteen patients (16 lesions) without pathologic evaluation were finally classified into the treatmentrelated changes group up to a median period of 7.5 months (range, 2-46 months). Patient characteristics are listed in Table 1.

\section{GSI quantitative parameters to differentiate between Glioma recurrence and treatment-related changes}

Table 2 enlists the differences in dual-energy spectral CT imaging quantitative parameters between glioma recurrence and treatment-related changes. Examination of precontrast $\lambda_{\mathrm{HU}}, \mathrm{Z}_{\mathrm{eff}}, \mathrm{Z}_{\mathrm{eff-N}}, \mathrm{IC}, \mathrm{IC}_{\mathrm{N}}$, and venous phase $\mathrm{IC}_{\mathrm{N}}$ $(P>0.05)$ on dual-energy spectral CT images showed no significant differences in quantitative parameters. The mean $\lambda_{\mathrm{HU}}(P<0.001)$ for glioma recurrence was $1.426 \pm$ 0.762 vs. $0.314 \pm 0.373$ for treatment-related changes in the venous phase. In addition, the $Z_{\text {eff }}(P<0.001)$ for glioma recurrence was $8.034 \pm 0.238$ vs. $7.671 \pm 0.151$ for treatment-related changes in the venous phase. Similarly, the $Z_{\text {eff-N }}(P<0.001)$ for glioma recurrence was $1.058 \pm$ 0.020 vs. $1.013 \pm 0.024$ for treatment-related changes. The IC $(P<0.001)$ for glioma recurrence was $7.319 \pm 3.967 \mathrm{vs}$. $1.703 \pm 2.049$ for treatment-related changes in the venous

Table 1 Patient characteristics

\begin{tabular}{ll}
\hline Characteristic & Value \\
\hline Age (mean years) & $39.3 \pm 13.0$ \\
Sex (No. of patients) (\%) & $13(46.4)$ \\
Male & $15(53.6)$ \\
Female & \\
WHO classification (No. of lesions) (\%) & $15(53.6)$ \\
Grade II & $7(25.0)$ \\
Grade III & $6(21.4)$ \\
Grade IV & \\
Primary treatment (No. of patients) (\%) & $3(10.7)$ \\
Operation & $5(17.9)$ \\
Operation + radiation therapy & $20(71.4)$ \\
Operation + radiation therapy + chemotherapy & \\
Final diagnosis (No. of lesions) (\%) & $11(39.3)$ \\
Recurrence & $5(17.9)$ \\
Pathologic & $6(21.4)$ \\
Clinicoradiologic follow up & $17(60.7)$ \\
Treatment related changes & $2(7.1)$ \\
Pathologic & $15(53.6)$ \\
Clinicoradiologic follow up &
\end{tabular}


Table 2 Difference of GSI quantitative parameters between glioma recurrence and treatment-related changes

\begin{tabular}{llll}
\hline GSI quantitative parameters & glioma recurrence & treatment related changes & $P$ Value \\
\hline Precontrast $\lambda \mathrm{HU}$ & $-0.007(-0.477,0.494)$ & $-0.064(-0.619,0.310)$ & 0.859 \\
Precontrast Zeff & $7.545(7.353,7.745)$ & $7.520(7.295,7.653)$ & 0.723 \\
Precontrast Zeff-N & $1.007(0.996,1.012)$ & $1.005(0.997,1.012)$ & 0.965 \\
Precontrast IC & $-0.108(-2.598,2.649)$ & $-0.375(-3.33,1.428)$ & 0.790 \\
Precontrast ICN & $0.733(0.509,1.102)$ & $0.969(0.504,1.086)$ & 0.723 \\
Venous phase $\lambda \mathrm{HU}$ & $1.426 \pm 0.762$ & $0.314 \pm 0.373$ & $<.001$ \\
Venous phase Zeff & $8.034 \pm 0.238$ & $7.671 \pm 0.151$ & $<0.001$ \\
Venous phase Zeff-N & $1.058 \pm 0.020$ & $1.013 \pm 0.024$ & $<0.001$ \\
Venous phase IC & $7.319 \pm 3.967$ & $1.703 \pm 2.049$ & $<0.001$ \\
Venous phase ICN & $0.636(-2.140,3.514)$ & $0.827(-0.0634,1.740)$ & 0.832 \\
\hline
\end{tabular}

All $P$ values for group comparisons were obtained by $t$ test

Data in parentheses are medians with interquartile range

phase (Fig. 3). The optimal venous phase $\lambda_{\mathrm{HU}}, \mathrm{Z}_{\text {eff, }} \mathrm{Z}_{\text {eff-N, }}$, and IC threshold was $1.03,7.75,1.04$, and $2.85 \mathrm{mg} / \mathrm{cm}^{3}$, achieving a sensitivity of $66.7,91.7,83.3$, and $91.7 \%$; specificity of 100.0, 77.8, 88.9, and 77.8\%; PPV of 100.0, 73.3, 83.3, and 73.3\%; NPV of 81.8, 93.3, 88.9, and 93.3\%; and accuracy of $86.7,83.3,86.7$, and $83.3 \%$, respectively (Table 3). The respective AUCs were 0.912, 0.912, 0.931, and 0.910 in glioma recurrence and treatment-related changes (Fig. 4).

\section{Discussion}

A high incidence of treatment-related changes has been noted in patients who undergo post-operative radiotherapy or combined chemoradiotherapy with temozolomide. Moreover, routinely available CT and MRI techniques do not allow a reliable distinction between glioma recurrence and treatment-related changes $[1,14]$. Moreover, the presence of a new contrast-enhanced lesion during follow-up

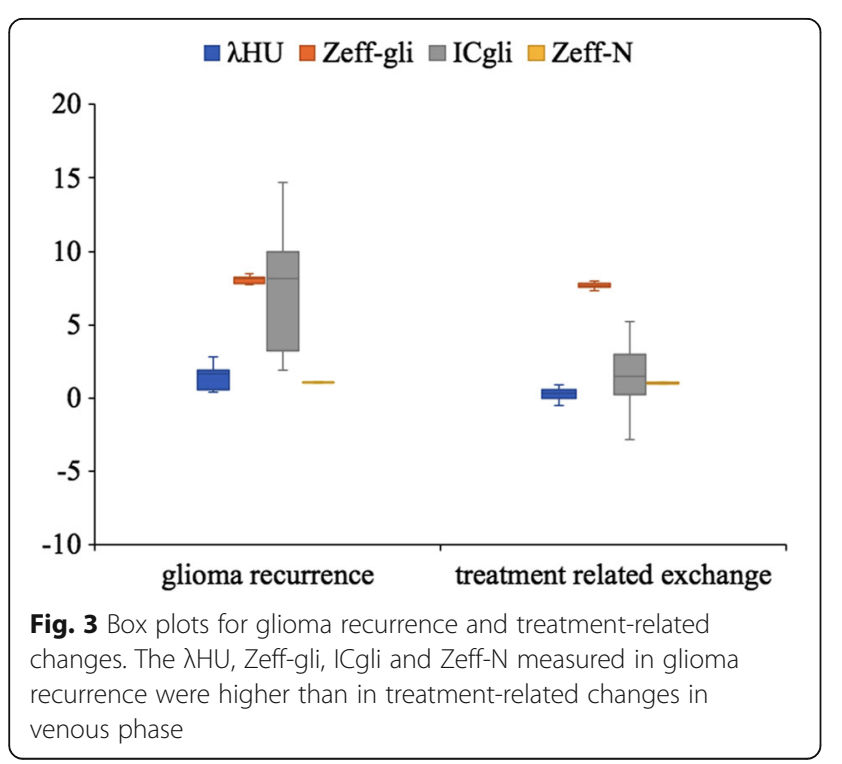

imaging typically indicates a mixture of necrotic tissue and progressive tumor growth; this adds to the overall complexity of lesion characterization [3].

In this study, we used quantitative parameters measured on dual-energy spectral CT to differentiate between glioma recurrence and treatment-related changes. Additionally, the slope of $\lambda_{\mathrm{HU}}, \mathrm{Z}_{\text {eff, }} \mathrm{Z}_{\text {eff-N }}$, and IC in the venous phase was higher in patients with glioma recurrence than in those with treatment-related changes.

The $\lambda_{\mathrm{HU}}$ value was automatically generated for the given ROIs, describing the dynamic changes of measured CT Hounsfield units of ROIs against increasing keV values within the range of 40 to $140 \mathrm{keV}$ [10]. In our study, we calculated $\lambda_{\mathrm{HU}}$ as the difference between the CT value on $40 \mathrm{keV}$ and $70 \mathrm{keV}$ divided by the energy difference $(30 \mathrm{keV})$. Our results showed that the venous phase $\lambda_{\mathrm{HU}}$ in glioma recurrence was higher than in treatment-related changes, indicative of feasibility of enhancing venous phase $\lambda_{\mathrm{HU}}$ as a differentiating factor. The ROC analysis in our study revealed that the venous phase $\lambda_{\mathrm{HU}}$ was highly specific (100\%) for differentiating glioma recurrence from treatment-related changes. These findings were similar to the findings in previous reports $[10,13]$. Srinivasan et al. also reported that spectral HU curve is a potentially useful parameter to differentiate between benign and malignant neck pathologic findings [15].

$Z_{\text {eff }}$ is also a quantitative index for characterization of composition of a nodule. Furthermore, it signifies the composite atom in a compound or mixture of various materials and is important in the prediction of X-rays' interaction with a substance [10]. According to our study results, venous phase $Z_{\text {eff }}$ and $Z_{\text {eff-N }}$ were higher in glioma recurrence than in treatment-related changes, which was indicative of the feasibility of venous phase $Z_{\text {eff }}$ and $Z_{\text {eff- } N}$ as a differentiating factor; these results are consistent with the findings in previous reports $[10,13]$. 
Table 3 GSI quantitative parameters for differential diagnosis of glioma recurrence and treatment-related changes

\begin{tabular}{|c|c|c|c|c|c|c|c|c|}
\hline $\begin{array}{l}\text { GSI Quantitative } \\
\text { Parameters }\end{array}$ & $A \cup C$ & $\begin{array}{l}\text { Maximum } \\
\text { Youden } \\
\text { Index }\end{array}$ & $\begin{array}{l}\text { Threshold } \\
\text { of } \\
\text { Parameters }\end{array}$ & Sensitivity (\%) & Specificity (\%) & PPV (\%) & NPV (\%) & Accuracy \\
\hline $\begin{array}{l}\text { Venous phase } \\
\lambda H U\end{array}$ & $0.912(0.812,1.012)$ & 0.667 & 1.03 & $66.7(56.7,76.7)$ & $100.0(90.0,110.0)$ & $100.0(90.0,110.0)$ & 81.8 (71.8.91.8) & $86.7(76.7,96.7)$ \\
\hline $\begin{array}{l}\text { Venou phase } \\
\text { Zeff }\end{array}$ & $0.912(0.810,1.014)$ & 0.695 & 7.75 & $91.7(81.5101 .9)$ & $77.8(67.6,88.0)$ & $73.3(63.1,83.5)$ & $93.3(83.1103 .5)$ & $83.3(73.1,93.5)$ \\
\hline $\begin{array}{l}\text { Venous phase } \\
\text { Zeff-N }\end{array}$ & $0.931(0.843,1.019)$ & 0.722 & 1.04 & $83.3(74.5,92.1)$ & $88.9(80.1,97.7)$ & $83.3(74.5,92.1)$ & $88.9(80.1,97.7)$ & $86.7(77.9,95.5)$ \\
\hline $\begin{array}{l}\text { Venous phase } \\
\text { IC }\end{array}$ & $0.910(0.810,1.010)$ & 0.695 & 2.85 & $91.7(81.9101 .5)$ & $77.8(68.0,87.6)$ & $73.3(63.5,83.1)$ & $93.3(83.5103 .1)$ & $83.3(73.5,93.1)$ \\
\hline
\end{tabular}

The results of our ROC analysis showed that the venous phase $Z_{\text {eff }}$ was highly sensitive in differentiating glioma recurrence from treatment-related changes.

$\mathrm{Lv}$ et al. reported a linear relationship between the measured and actual iodine concentrations in their study upon testing tubes filled with known iodine concentrations and iodine concentrations measured from the iodine-based material-decomposition images [9]. Our study results showed that venous phase IC was higher in glioma recurrence than in treatment-related changes, thereby suggesting the potential of venous phase IC as a differentiating factor. The ROC analysis in our study revealed that the venous phase IC was highly sensitive for differentiating glioma recurrence from treatment-related changes. A previous report also suggested the usefulness of IC in thyroid nodules as a quantitative parameter to distinguish between malignant and benign nodules [10].
Furthermore, measured IC in lesions might be a useful quantitative parameter of the lesion's blood supply $[11,12]$. Moding et al. showed that dual energy CT is a powerful tool for monitoring vascular changes after radiation therapy [16]. Increased IC could also be attributed to changes in tumor-associated vascular patterns and an increased blood supply [17].

Our study showed no significant differences with respect to venous phase $\mathrm{IC}_{\mathrm{N}}$, contradicted with venous phase IC. This may likely be because of the sample size being relatively small, and the fact that gliomas are a heterogeneous group of tumors, which sometimes showed up as poor soft tissue contrast on dual-energy spectral CT, leading to potential selection bias.

There are a few other limitations to this study. In our experience, the differential diagnosis of lesions in the vicinity of the skull base is rather challenging given the

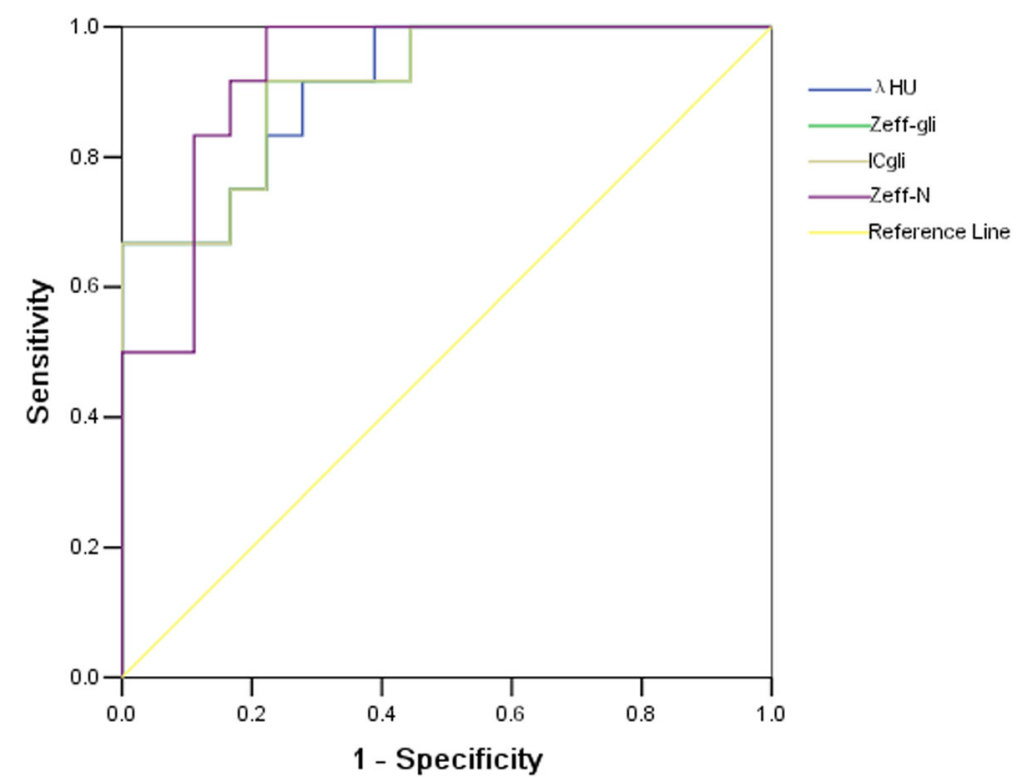

Fig. 4 Graphs show receiver operating characteristic curves of $\lambda H U$, Zeff-gli, ICgli and Zeff-N in venous phase for differentiating glioma recurrence from treatment-related changes in patients. The venous Zeff-N had the highest AUC (0.931), with the optimal threshold of 1.04 AUC $=$ area under the curve 
presence of many small blood vessels on the cerebral cortex; this might have led to inaccuracies in differential diagnosis. Second, it should be noted that all gliomarecurrence lesions in this study were not analyzed by biopsy; some were confirmed by follow-up evaluations. This may have influenced the study results. Third, relevant data on interobserver reliability are lacking, because images were assessed in consensus. Finally, tumor heterogeneity and spatial heterogeneity were not considered in this study. Hence, further large-scale prospective trials, with glioma classification and tumor heterogeneity are required to validate our results by dual-energy spectral imaging.

\section{Conclusions}

Dual energy GSI-CT may potentially afford quantitative values to help differentiate between glioma recurrence and treatment-related changes. Thus, a dual-energy spectral CT would mean a second examination in addition to the routine MRI in clinical practice.

\section{Abbreviations}

GSI: gemstone spectral imaging; IC: iodine concentration; $I_{N}$ : normalized iodine concentration; $Z_{\text {eff: }}$ effective atomic number; $Z_{\text {eff-N: }}$ normalized effective atomic number; $\lambda_{H U}$ : the slope of the spectral Hounsfield unit curve

\section{Acknowledgements}

Not applicable.

\section{Authors' contributions}

$Y L, J Z, Y C$ and $Z C$ conceived and designed research; $X L, L T, H H, Z L, Y W, L H$, $M S$, and YY collected data and conducted research; CG, CL, RZ, and CX analyzed and interpreted data; JZ wrote the initial paper; $Z C$ revised the paper; YL and ZC had primary responsibility for final content. All authors read and approved the final manuscript.

\section{Funding}

This study was funded by National Basic Research Program of China (973 Program, grant number 2015CB755500); Science and Technology Planning Project of Guangdong Province, China (grant number 2012B031800102, 2016A020213004); Guangzhou Science and Technology Program key projects (grant number 201803010056); National Natural Science Foundation of China (grant number 81572500); and Hunan Young Talents (grant number 2016RS3036). All of them play a role in the design of the study and collection, analysis, and interpretation of data and in writing the manuscript.

\section{Availability of data and materials}

The datasets generated and analyzed during the current study are available from the corresponding author on reasonable request.

\section{Ethics approval and consent to participate}

The written consents were obtained from the patients or the relatives of patients. The study was approved by the Ethics Committee of the Sun Yatsen University Cancer Center.

\section{Consent for publication}

All data published here are under the consent for publication.

\section{Competing interests}

The authors declare that they have no competing interests.

\section{Author details}

'Department of Medical Imaging, Sun Yat-sen University Cancer Center, State Key Laboratory of Oncology in South China, Collaborative Innovation Center for Cancer Medicine, 651 Dongfeng Road East, Guangzhou 510060, China.
${ }^{2}$ Department of head and neck oncology, Phase 1 clinical trial ward, The cancer center of the fifth affiliated hospital of Sun Yat-sen University, Zhuhai 519001, China. ${ }^{3}$ Department of Radiology, Shantou Central Hospital, No.114 Waima Road, Shantou 515041, Guangdong, China. ${ }^{4}$ Department of Neurosurgery/Neuro-oncology, Sun Yat-sen University Cancer Center, State Key Laboratory of Oncology in South China, Collaborative Innovation Center for Cancer Medicine, 651 Dongfeng Road East, Guangzhou 510060, China.

Received: 15 September 2019 Accepted: 24 December 2019

Published online: 16 January 2020

\section{References}

1. Brandsma D, Stalpers L, Taal W, Sminia P, van den Bent MJ. Clinical features, mechanisms, and management of pseudoprogression in malignant gliomas. Lancet Oncol. 2008;9:453-61.

2. Zhang $H, M a L$, Wang $Q$, Zheng $X$, Wu C, Xu B. Role of magnetic resonance spectroscopy for the differentiation of recurrent glioma from radiation necrosis: a systematic review and meta-analysis. Eur J Radiol. 2014;83:2181-9.

3. Verma N, Cowperthwaite MC, Burnett MG, Markey MK. Differentiating tumor recurrence from treatment necrosis: a review of neuro-oncologic imaging strategies. Neuro-Oncology. 2013;15:515-34.

4. Chao ST, Ahluwalia MS, Barnett GH, Stevens GHJ, Murphy ES, Stockham AL, et al. Challenges with the diagnosis and treatment of cerebral radiation necrosis. Int J Radiat Oncol Biol Phys. 2013:87:449-57.

5. Enslow MS, Zollinger LV, Morton KA, Kadrmas DJ, Butterfield RI, Christian PE, et al. Comparison of 18F-fluorodeoxyglucose and 18F-fluorothymidine PET in differentiating radiation necrosis from recurrent glioma. Clin Nucl Med. 2012;37:854-61.

6. Pyka T, Gempt J, Ringel F, Hüttinger S, van Marwick S, Nekolla S, et al. Prediction of glioma recurrence using dynamic ${ }^{18} \mathrm{~F}$-fluoroethyltyrosine PET. AJNR Am J Neuroradiol. 2014;35:1924-9.

7. Shah R, Vattoth S, Jacob R, Manzil FF, O'Malley JP, Borghei P, et al. Radiation necrosis in the brain imaging features and differentiation from tumor recurrence. Radiographics. 2012;32:1343-59.

8. Kim HS, Goh MJ, Kim N, Choi CG, Kim SJ, Kim JH. Which combination of MR imaging modalities is best for predicting recurrent glioblastoma? Study of diagnostic accuracy and reproducibility. Radiology. 2014;273:831-43.

9. Lv P, Lin XZ, Li J, Li W, Chen K. Differentiation of small hepatic hemangioma from small hepatocellular carcinoma: recently introduced spectral CT method. Radiology. 2011;259:720-9.

10. Li M, Zheng X, Li J, Yang Y, Lu C, Xu H, et al. Dual-energy computed tomography imaging of thyroid nodule specimens comparison with pathologic findings. Investig Radiol. 2012;47:58-64.

11. Zhang XF, Lu Q, Wu LM, Zou AH, Hua XL, Xu JR. Quantitative iodine-based material decomposition images with spectral $C T$ imaging for differentiating prostatic carcinoma from benign prostatic hyperplasia. Acad Radiol. 2013;20: $947-56$.

12. Yu Y, Lin $X$, Chen $K$, Chai W, Hu S, Tang $R$, et al. Hepatocellular carcinoma and focal nodular hyperplasia of the liver: differentiation with $C T$ spectral imaging. Eur Radiol. 2013;23:1660-8.

13. Liu X, Ouyang D, Li H, Zhang R, Lv Y, Yang A, et al. Papillary thyroid cancer: dual-energy spectral $C T$ quantitative parameters for preoperative diagnosis of metastasis to the cervical lymph nodes. Radiology. 2015;275:167-76.

14. Jena A, Taneja S, Jha A, Damesha NK, Negi P, Jadhav GK, et al. Multiparametric evaluation in differentiating glioma recurrence from treatment-induced necrosis using simultaneous ${ }^{18}$ F-FDG-PET/MRI: a singleinstitution retrospective study. AJNR Am J Neuroradiol. 2017;38:899-907.

15. Srinivasan A, Parker RA, Manjunathan A, Ibrahim M, Shah GV, Mukherji SK. Differentiation of benign and malignant neck pathologies preliminary experience using spectral computed tomography. J Comput Assist Tomogr. 2013;37:666-72.

16. Moding EJ, Clark DP, Qi Y, Li Y, Ma Y, Ghaghada K, et al. Dual-energy microcomputed tomography imaging of radiation-induced vascular changes in primary mouse sarcomas. Int J Radiat Oncol Biol Phys. 2013;85:1353-9.

17. Vogl TJ, Schulz B, Bauer RW, Stöver T, Sader R, Tawfik AM. Dual-energy CT applications in head and neck imaging. AJR Am J Roentgenol. 2012;199:S34-9.

\section{Publisher's Note}

Springer Nature remains neutral with regard to jurisdictional claims in published maps and institutional affiliations. 